\title{
ALTERNATING TRILINEAR FORMS AND \\ GROUPS OF EXPONENT 6
}

\author{
Dedicated to the memory of Hanna Neumann
}

M. D. ATKINSON

(Received 29 May 1972)

Communicated by M. F. Newman

The theory of alternating bilinear forms on finite dimensional vector spaces $V$ is well understood; two forms on $V$ are equivalent if and only if they have equal ranks. The situation for alternating trilinear forms is much harder. This is partly because the number of forms of a given dimension is not independent of the underlying field and so there is no useful canonical description of an alternating trilinear form.

In this paper we consider the set of all alternating trilinear forms on all finite dimensional vector spaces over a fixed finite field $F$ and show that this set has a certain finiteness property. We then give a brief description of how this result may be used to prove two theorems on varieties of groups; in particular, that every group of exponent 6 has a finite basis for its laws. The details may be found in my D. Phil. thesis [1] which was written while I held a scholarship from the Science Research Council. This research was supervised by Dr. P. M. Neumann and Professor G. Higman for whose help I am heartily grateful.

\section{Preliminaries}

Throughout this paper $F$ will denote a finite field with $q$ elements. A finite dimensional vector space over $F$ on which is defined an alternating trilinear form $(u, v, w)$ is said to be a $T$-space (over F). If $V$ is a $T$-space and $U \leqq V$ then the restriction of the alternating trilinear form on $V$ to $U$ gives $U$ the structure of a $T$-space and we sometimes call attention to this by saying that $U$ is a sub-T-space of $V$. A linear transformation $\alpha: V \rightarrow U$, where $V$ and $U$ are $T$-spaces, is said to be a homomorphism if, for all $u, v, w \in V,(u \alpha, v \alpha, w \alpha)=(u, v, w)$. The terms isomorphism, epimorphism and monomorphism are defined in the obvious way.

If $A, B, C$ are subsets of the $T$-space $V$ we shall write $(A, B, C)=0$ if $(a, b, c)=0$ for all $a \in A, b \in B, c \in C$. We write $(v, A, B)=0$ if $(\{v\}, A, B)=0$. 
Obviously, $\{v \in V \mid(v, V, V)=0\}$ is a subspace of $V$; we shall call it the singular part of $V$. It is easy to see that all vector space complements for the singular part of $V$ are isomorphic (as $T$-spaces) and so we may unambiguously refer to any one of them as the non-singular part of $V$. A $T$-space is said to be non-singular if it coincides with its non-singular part and totally singular if it coincides with its singular part.

If $U$ and $V$ are $T$-spaces then we may conside their direct sum $U \oplus V$ in the usual sense of linear algebra. Of course, this will not have the structure of a $T$-space unless the values of $\left(u_{1}, v_{1}, v_{2}\right)$ and $\left(u_{1}, u_{2}, v_{1}\right)$ are defined for all $u_{1}, u_{2} \in U$ and $v_{1}, v_{2} \in V$ : e.g. these values are defined if $U$ and $V$ are subspaces of some $T$-space $W$. If $(U, U, V)=(U, V, V)=0$ then we write $U \oplus V=U \oplus_{c} V$. Inductively we define $U_{1} \oplus_{c} U_{2} \oplus_{c} \cdots \oplus_{c} U_{n}$ as $\left(U_{1} \oplus_{c} \cdots \oplus_{c} U_{n-1}\right) \oplus_{c} U_{n}$ and write $U^{n}$ for this $T$-space if all the $U_{i}$ are isomorphic to some fixed $T$-space $U$.

Let $\mathfrak{I}$ be the set of all (isomorphism classes of) $T$-spaces. We define a partial order $\preccurlyeq$ on $\mathfrak{I}$ by defining $U \preccurlyeq V$ if $U$ is isomorphic to a sub- $T$-space of $V$. Our main result on $T$-spaces is that, with respect to this ordering, $\mathfrak{I}$ is a partially well-ordered set. $\mathfrak{I}$ is partially well-ordered if and only if its closed subsets satisfy the minimum condition under inclusion; a subset $\mathfrak{X}$ of $\mathfrak{I}$ is said to be closed if, whenever $V \in \mathfrak{X}$ and $U \preccurlyeq V, U \in \mathfrak{X}$. The closure of a set $\mathfrak{X}$, cl $\mathfrak{X}$, is $\{V \mid V \preccurlyeq U \in \mathfrak{X}\}$.

If $A$ and $B$ are subsets of the $T$-space $V$ then $\{v \in V \mid(v, A, B)=0\}$ is denoted by $\operatorname{Ann}(A, B)$ and it is obviously a subspace of $V$.

LEMMA 1.1. If $A$ and $B$ are subspaces of the T-space $V$ then

$$
[V: \operatorname{Ann}(A, B)] \leqq(\operatorname{dim} A) \cdot(\operatorname{dim} B) .
$$

Proof. Let $e_{1}, \cdots, e_{a}$ and $f_{1}, \cdots, f_{b}$ be bases for $A$ and $B$ respectively. Clearly, $\operatorname{Ann}(A, B)=\bigcap_{\substack{i=1 . a \\ j=1 . b}} \operatorname{Ann}\left(e_{i}, f_{j}\right)$. However, $\operatorname{Ann}\left(e_{i}, f_{j}\right)$ is the kernel of the linear functional $x \mapsto\left(x, e_{i}, f_{j}\right)$ and so has codimension at most 1 in $V$.

LemMa 1.2. Let $V$ be a T-space of dimension at least $r^{2}$. Then $V$ has a totally singular subspace of dimension $r$.

Proof. We proceed by induction on $r$, the case $r=0$ being trivial. Let $r \geqq 1$ and suppose that the lemma holds with $r-1$ in place of $r$. Then there exists a totally singular subspace $S$ of dimension $r-1$. Since, by Lemma 1.1, $[V: \operatorname{Ann}(S, S)] \leqq(r-1)^{2}$ we see that $\operatorname{Ann}(S, S) \nsubseteq S$ and so there exists $u \in \operatorname{Ann}(S, S)-S$. Obviously, $\langle S, u\rangle$ is totally singular and of dimension $r$.

If $x$ is a fixed element of some $T$-space $V$ and $U \leqq V$ then $\left(x, u_{1}, u_{2}\right)$ with $u_{1}, u_{2} \in U$ provides an alternating bilinear form on $U$. The rank of this form is called the rank of $x$ on $U$. In this context we recall a simple fact about alternating bilinear forms. 
LEMMA 1.3. Let $(x, y)$ be an alternating bilinear form of rank $r$ defined on a vector space $V$ and let $U$ be a subspace of $V$ of codimension $n$. Then the restriction of the form to $U$ has rank at least equal to $r-2 n$.

\section{Some basic lemmas}

If $e_{1}, \cdots, e_{n}$ is some given basis for a $T$-space $V$ the scalars $\left(e_{i}, e_{j}, e_{k}\right)$, for all $1 \leqq i, j, k \leqq n$, are called basic products. To define a $T$-space uniquely it is sufficient to give a basis $e_{1}, \cdots, e_{n}$ and basic products $\left(e_{1}, e_{j}, e_{k}\right)$, for all $1 \leqq i<j$ $<k \leqq n$.

For each $n$ we define a certain $T$-space $V_{n}$ by a basis $x, a_{1}, a_{2}, \cdots, a_{2 n}$ together with the basic products

$$
\begin{aligned}
\left(x, a_{2 i-1}, a_{2 i}\right) & =-\left(x, a_{2 i}, a_{2 i-1}\right)=.1 \text { for } i=1, \cdots, n \\
\left(x, a_{i}, a_{j}\right) & =0 \text { for all other } i, j \\
\left(a_{i}, a_{j}, a_{k}\right) & =0 \text { for all } i, j, k .
\end{aligned}
$$

We observe that $V_{n}$ has a totally singular subspace of codimension 1 and that the rank of $x$ on this subspace is as large as possible. We note that $V_{1}$ is the unique minimal non-singular element of $\mathfrak{I}$.

LEMMA 2.1. If the T-space $U$ is non-singular then every homomorphism of $U$ into a $T$-space $V$ is a monomorphism.

Proof. Let $\alpha: U \rightarrow V$ be a homomorphism and let $x$ belong to the kernel of $\alpha$. Then $x \alpha=0$ and so, for all $u, v \in U,(x \alpha, u \alpha, v \alpha)=0$. Thus, for all $u, v \in U$, $(x, u, v)=0$ and so $x=0$ as $U$ is non-singular.

THEOREM 2.2. For every $T$-space $V$ there exists an integer $n$ such that $V \preccurlyeq V_{1}^{n}$.

Proof. If $\pi$ is a monomorphism from the non-singular part of $V$ into some $V_{1}^{n}$ then it is easy to see that $\pi$ can be extended to a monomorphism of $V$ into $V_{1}^{n+s}$ where $s$ is the dimension of the singular part of $V$. So, without loss in generality, we may assume that $V$ is non-singular.

Let $e_{1}, \cdots, e_{r}$ be a basis for $V$ and let $U_{1}, \cdots, U_{n}$ be all the subsets $\left\{e_{i}, e_{j}, e_{k}\right\}$ with $1 \leqq i<j<k \leqq r$. Thus, $n=\left(\begin{array}{l}r \\ 3\end{array}\right)$. Suppose that $V_{1}^{n}$ is defined by the basis $a_{1}, b_{1}, c_{1}, a_{2}, b_{2}, c_{2}, \cdots, a_{n}, b_{n}, c_{n}$ together with the basic products $\left(a_{i}, b_{i}, c_{i}\right)$ $=\left(b_{i}, c_{i}, a_{i}\right)=\left(c_{i}, a_{i}, b_{i}\right)=-\left(c_{i}, b_{i}, a_{i}\right)=-\left(b_{i}, a_{i}, c_{i}\right)=-\left(a_{i}, c_{i}, b_{i}\right)=1$, for all $1 \leqq i \leqq n$ and all other basic products are zero.

Define maps $\gamma_{m}: U_{m} \rightarrow V_{1}^{n}$ by $e_{i} \gamma_{m}=a_{m}, e_{j} \gamma_{m}=b_{m}, e_{k} \gamma_{m}=\left(e_{i}, e_{j}, e_{k}\right) c_{m}$ where $U_{m}=\left\{e_{i}, e_{j}, e_{k}\right\}$ with $i<j<k$. 
Now define a linear transformation $\alpha$ from $V$ into $V_{1}^{n}$ by defining it as follows on the basis vectors and extending it by linearity.

$$
e_{i} \alpha=e_{i 1}+\cdots+e_{i n} \text { where } e_{\cdot m}= \begin{cases}e_{i} \gamma_{m} & \text { if } e_{i} \in U_{m} \\ 0 & \text { if } e_{i} \notin U_{m}\end{cases}
$$

To complete the proof we only have to verify that $\left(e_{i} \alpha, e_{j} \alpha, e_{k} \alpha\right)=\left(e_{i}, e_{j}, e_{k}\right)$ for all $1 \leqq i<j<k \leqq r$ and then appeal to Lemma 2.1. Suppose that $U_{m}=\left\{e_{i}, e_{j}, e_{k}\right\}$ with $i<j<k$. Then $\left(e_{i} \alpha, e_{j} \alpha, e_{k} \alpha\right)$

$$
\begin{aligned}
& =\left(e_{i 1}+\cdots+e_{i n}, e_{j 1}+\cdots+e_{j n}, e_{k 1}+\cdots+e_{k n}\right) \\
& =\left(e_{i 1}, e_{j 1}, e_{k 1}\right)+\cdots+\left(e_{i n}, e_{j n}, e_{k n}\right) \text { by definition of } V_{1}^{n} .
\end{aligned}
$$

$\operatorname{But}\left(e_{i l}, e_{j l}, e_{k l}\right)$ is non-zero only when $e_{i l}, e_{j l}$ and $e_{k l}$ are all non-zero and this can only happen when $\left\{e_{i}, e_{j}, e_{k}\right\}=U_{l}$, i.e. when $l=m$.

$$
\text { Hence, } \begin{aligned}
\left(e_{i} \alpha, e_{j} \alpha, e_{k} \alpha\right) & =\left(e_{i m}, e_{j m}, e_{k m}\right)=\left(e_{i} \gamma_{m}, e_{j} \gamma_{m}, e_{k} \gamma_{m}\right) \\
& =\left(a_{m}, b_{m},\left(e_{i}, e_{j}, e_{k}\right) c_{m}\right)=\left(e_{i}, e_{j}, e_{k}\right)\left(a_{m}, b_{m}, c_{m}\right) \\
& =\left(e_{i}, e_{j}, e_{k}\right) .
\end{aligned}
$$

The following lemmas exploit our knowledge of alternating bilinear forms.

Lemma 2.3. Let $X$ be a vector space of alternating bilinear forms on the vector space $V$ and suppose that, for every $S$ in $X$, the rank of $S$ is less than $2 r$. $T$ hen $V$ has a subspace of codimension at most $r(r-1)$ on which every element of $X$ vanishes.

Proof. We prove the lemma by induction on $r$, the case $r=1$ being immediate. We may suppose that $X$ contains a form $S_{1}$ of rank precisely $2(r-1)$. Then $U_{1}=\left\{u \in V \mid S_{1}(u, v)=0\right.$ for all $\left.v \in V\right\}$ is of codimension $2(r-1)$. We shall show that, for every $S_{2}$ in $X$, the restriction of $S_{2}$ to $U_{1}$ has rank at most $2(r-2)$ on $U_{1}$. Suppose the contrary, so that some $S_{2}$ has rank $2(r-1)$ on $U_{1}$. Then $U_{2}=\left\{u \in V \mid S_{2}(u, v)=0\right.$ for all $\left.v \in V\right\}$ has codimension $2(r-1)$ in $V$ and $U_{1} \cap U_{2}$ has codimension $2(r-1)$ in $U_{1}$. Thus, we may choose $x_{1}^{(i)}, \cdots, x_{2(r-1)}^{(i)}$ as a basis for $U$ modulo $U_{1} \cap U_{2}$ and take the $x_{j}^{(i)}, i=1,2$, $j=1, \cdots, 2(r-1)$, as part of a basis for $V$, and have

$$
\begin{aligned}
& S_{1}\left(x_{2 j-1}^{(2)}, x_{2 j}^{(2)}\right)=-S_{1}\left(x_{2 j}^{(2)}, x_{2 j-1}^{(2)}\right)=1 \text { and } \\
& S_{2}\left(x_{2 j-1}^{(1)}, x_{2 j}^{(1)}\right)=-S_{2}\left(x_{2 j}^{(1)}, x_{2 j-1}^{(1)}\right)=1 \text { for all } 1 \leqq j \leqq r-1
\end{aligned}
$$

together with $S_{1}(x, y)=0$ and $S_{2}(x, y)=0$ for all other pairs of basis elements. It is then evident that $S_{1}+S_{2}$ has rank $4(r-1) \geqq 2 r$ (since $r>1$ ). This contradiction shows that $S_{2}$ has rank less than $2(r-1)$ on $U_{1}$. The inductive hypothesis now yields a subspace $U_{0}$ of $U_{1}$ of codimension at most $(r-1)(r-2)$ in $U_{1}$ on 
which every element of $X$ vanishes. However,

$$
\begin{aligned}
{\left[V: U_{0}\right]=} & {\left[V: U_{1}\right]+\left[U_{1}: U_{0}\right] \leqq 2(r-1)+(r-1)(r-2) } \\
& =r(r-1) .
\end{aligned}
$$

LEMMA 2.4. Let $S_{1}, \cdots, S_{r}$ be alternating bilinear forms on a vector space $V$ with the property that every non-zero linear combination $\sum_{i=1}^{r} \alpha_{i} S_{i}$ has rank at least $4 r(r-1)+2$. Then there exist $u_{1}, \cdots, u_{2 r} \in V$ such that the matrix

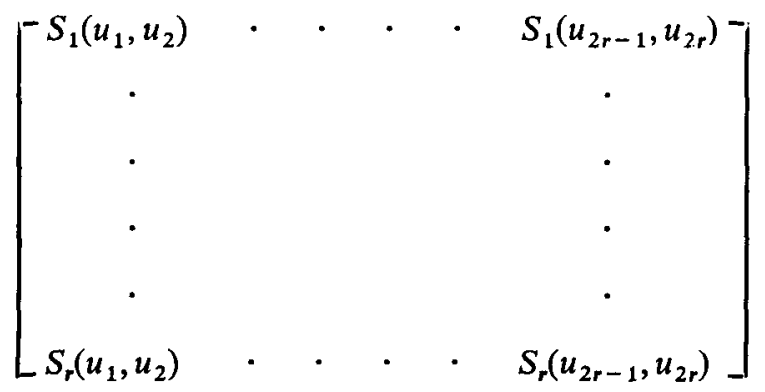

is non-singular and $S_{i}\left(u_{j}, u_{k}\right)=0$ for all other $i, j$ and $k$.

Proof. The lemma is proved by induction on $r$ being trivial if $r=1$. Assume that $r>1$ and that $S_{1}, \cdots, S_{r}$ satisfy the hypotheses of the lemma and that the lemma holds with $r-1$ in place of $r$. Then there exist $u_{1}, \cdots, u_{2 r-2}$ in $V$ such that the $r-1) \times(r-1)$ matrix

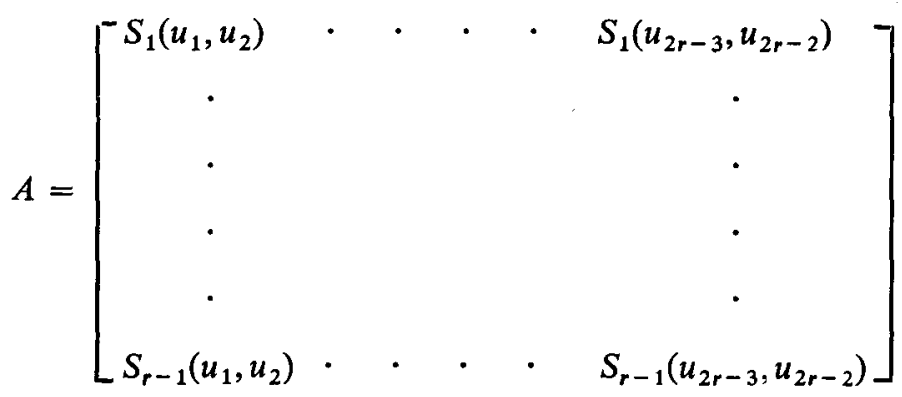

is non-singular and $S_{i}\left(u_{j}, u_{k}\right)=0$ for all other $i, j, k$ in $1 \leqq i \leqq r-1$ and $1 \leqq j$, $k \leqq 2 r-2$.

\section{Let}

$$
Y=\left\{y \in V \mid S_{i}\left(y, u_{j}\right)=0 \text { for all } 1 \leqq i \leqq r \text { and } 1 \leqq j \leqq 2 r-2\right\}
$$

which is a subspace of $V$ of codimension at most $2 r(r-1)$. To complete the inductive step it is only necessary to find $u_{2 r-1}, u_{2 r} \in Y$ so that the $r \times r$ matrix 


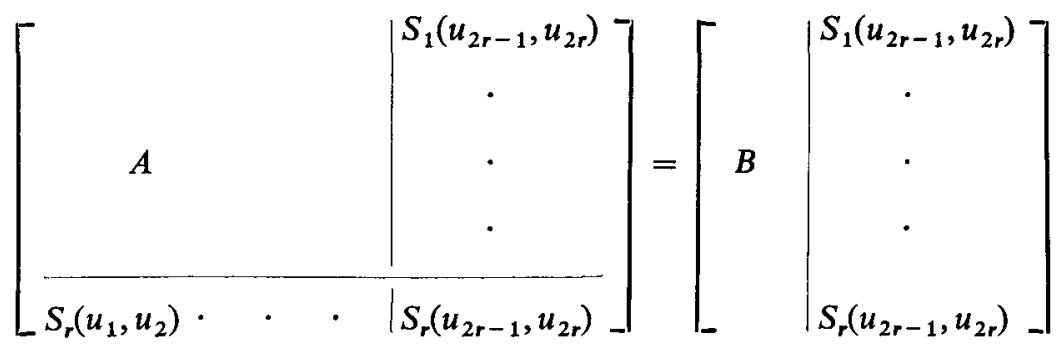

is non-singular. If, for every choice of $u_{2 r-1}, u_{2 r} \in Y$, this matrix is singular then, for every choice of $u_{2 r-1}, u_{2 r} \in Y$, there is a non-trivial dependence relation on $\rho_{1}, \cdots, \rho_{r}$, the rows of the matrix. However, the dependence relation must be the same (apart from scalar multiples) for all choices of $u_{2 r-1}, u_{2 r} \in Y$ because $B$ has row rank $r-1$ and so there is only one dependence relation on the rows of $B$. Therefore there exist scalars $\beta_{1}, \cdots \beta_{r}$ not all zero such that $\beta_{1} \rho_{1}+\cdots+\beta_{r} \rho_{r}=0$ no matter what elements $u_{2 r-1}, u_{2 r}$ are taken from $Y$. Hence $\beta_{1} S_{1}\left(u_{2 r-1}, u_{2 r}\right)$ $+\cdots+\beta_{r} S_{r}\left(u_{2 r-1}, u_{2 r}\right)=0$ for all $u_{2 r-1}, u_{2 r} \in Y$. But, by Lemma 1.3, $\sum_{i=1}^{r} \beta_{i} S_{i}$ has rank on $Y$ at least equal to $4 r(r-1)+2-2(2 r(r-1))>0$, which is a contradiction.

LEMMA 2.5. Let $S_{1}, \cdots, S_{r}$ be alternating bilinear forms on a vector space $V$ having the property that every non-zero linear combination $\sum_{i=1}^{r} \alpha_{i} S_{\text {, has rank }}$ at least $4 r(r-1)+2$. Then there exist $u, v \in V$ such that $S_{1}(u, v)=1$ and $S_{j}(u, v)=0$ for all $2 \leqq j \leqq r$.

Proof. The conditions of Lemma 2.4 are satisfied and there exist elements $u_{1}, \cdots u_{r}, v_{1}, \cdots v_{r}$ of $V$ such that the matrix

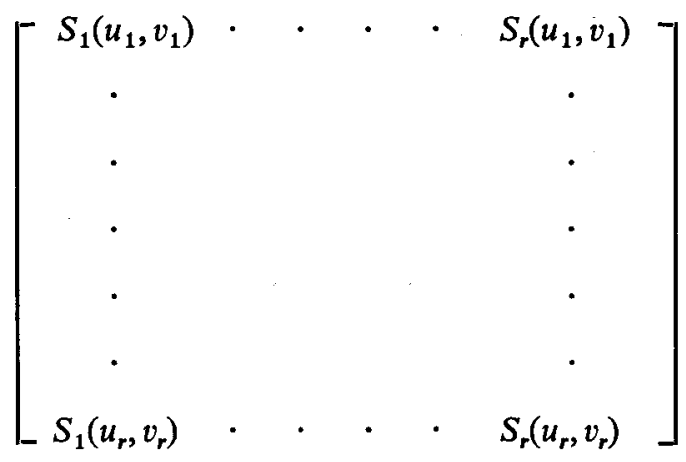

is non-singular and $S_{i}\left(u_{j}, v_{k}\right)=0$ if $j \neq k$. If $\rho_{1}, \cdots \rho_{r}$ are the rows of this matrix then, regarded as coordinate vectors, they span an $r$-dimensional vector space and one can find elements $\beta_{1}, \cdots \beta_{r}$ of $F$ such that

$$
\beta_{1} \rho_{1}+\cdots+\beta_{r} \rho_{r}=(1,0, \cdots, 0) \text {. }
$$


Therefore, $\beta_{1} S_{j}\left(u_{1}, v_{1}\right)+\cdots+\beta_{r} S_{j}\left(u_{r}, v_{r}\right)=\left\{\begin{array}{l}1 \text { if } j=1 \\ 0 \text { if } 2 \leqq j \leqq r\end{array}\right.$

However, since $S_{i}\left(u_{j}, v_{k}\right)=0$ if $j \neq k$,

$$
\beta_{1} S_{j}\left(u_{1}, v_{1}\right)+\cdots+\beta_{r} S_{j}\left(u_{r}, v_{r}\right)=S_{j}\left(\beta_{1} u_{1}+\cdots+\beta_{r} u_{r}, v_{1}+\cdots+v_{r}\right)
$$

and therefore we may put $u=\Sigma_{i=1}^{r} \beta_{i} u_{i}$ and $v=\sum_{i=1}^{r} v_{i}$ to prove the lemma.

Definition. Let $P$ be an $r$-dimensional $T$-space with a basis $x^{(1)}, \cdots, x^{(r)}$. We define the $T$-space $V\left(n, r, P, x^{(1)}, \cdots, x^{(r)}\right)$ by a basis

$$
\left\{x^{(1)}, \cdots, x^{(r)}\right\} \cup\left\{a_{i}^{(j)}, b_{i}^{(j)} \mid 1 \leqq i \leqq n, 1 \leqq j \leqq r\right\}
$$

together with the following basic products:

1) $\left(x^{(i)}, x^{(j)}, x^{(k)}\right)$; these are determined by $P$

2) $\left(x^{(i)}, x^{(j)}, a_{\mathrm{k}}^{(l)}\right)=\left(x^{(i)}, x^{(j)}, b_{\mathrm{k}}^{(l)}\right)=0$

3) $\left(x^{(i)}, a_{j}^{(l)}, a_{k}^{(m)}\right)=\left(x^{(i)}, b_{j}^{(l)}, b_{k}^{(m)}\right)=0$

4) $\left(x^{(i)}, a_{j}^{(l)}, b_{k}^{(m)}\right)=\delta_{i l} \delta_{l m} \delta_{j k}$

in each case the subscripts and superscripts running through all possible values, and

5) basic products which are determined by

$$
\left\langle a_{i}^{(j)}, b_{i}^{(j)} \mid 1 \leqq i \leqq n, 1 \leqq j \leqq r\right\rangle \text { being totally singular. }
$$

In the special case that $P$ is totally singular $V\left(n, r, P, x^{(1)}, \cdots, x^{(r)}\right)$ is isomorphic to $V_{n}^{r}$, the $i$ th central direct summand being generated by $x^{(i)}, a_{1}^{(i)}, b_{1}^{(i)}$, $\cdots, a_{n}^{(i)}, b_{n}^{(i)}$.

LEMMA 2.6. Suppose that $V$ is a T-space with a totally singular subspace $V_{0}$ and an r-dimensional subspace $P$. Suppose that the rank of every non-zero element of $P$ on $V_{0}$ is at least $8 n^{2} r^{2}+4 r^{2}$.Then $V$ has a sub-T-space isomorphic to $V\left(n, r, P, x^{(1)}, \cdots, x^{(r)}\right)$ where $x^{(1)}, \cdots, x^{(r)}$ is any basis for $P$.

Proof. We construct the required subspace by finding elements of $V_{0}$

$$
a_{1}^{(1)}, b_{1}^{(1)}, \cdots, a_{1}^{(r)}, b_{1}^{(r)}, a_{2}^{(1)}, b_{2}^{(1)}, \cdots, a_{2}^{(r)}, b_{2}^{(r)}, \cdots, a_{n}^{(r)}, b_{n}^{(r)}
$$

in that order, which satisfy the conditions of the above definition. Suppose that we have successfully found $a_{i}^{(j)}, b_{i}^{(j)}$ for all $1 \leqq i<m \leqq n$ and $1 \leqq j \leqq r$ to construct some subspace $U$ isomorphic to $V\left(m-1, r, P, x^{(1)}, \cdots, x^{(r)}\right)$. Let $U_{0}=V_{0} \cap \operatorname{Ann}(U, U)$. This has codimension at most $4 m^{2} r^{2}$ in $V_{0}$ and hence every non-zero element of $P$ has, by Lemma 1.3 , rank on $U_{0}$ at least $8 n^{2} r^{2}+4 r^{2}$ $-8 m^{2} r^{2} \geqq 4 r^{2}$ since $m \leqq n$. Now we can, by Lemma 2.5 , choose $a_{m}^{(1)}, b_{m}^{(1)} \in U_{0}$ 
to satisfy the correct conditions. Similarly, we can construct $a_{m}^{(2)}, b_{m}^{(2)}, \cdots, a_{m}^{(r)}, b_{m}^{(r)}$, $\cdots, a_{n}^{(r)}, b_{n}^{(r)}$.

LEMMA 2.7. Let $V$ be a vector space on which is defined a function $\beta$ taking non-negative integral values and satisfying
a) $\beta(x+y) \leqq \beta(x)+\beta(y)$ and
b) $\beta(\lambda x)=\beta(x)$

for all $x, y \in V$ and $\lambda \in F-\{0\}$. Suppose that every m-dimensional subspace of $V$ contains a non-zero vector $v$ such that $\beta(v) \leqq n$. Then $V$ has a subspace $U$ of codimension $m-1$ such that

$$
\beta(u) \leqq 2 m n \text { for all } u \in U .
$$

Proof. Clearly, it is possible to find a subspace $U$ of codimension $m-1$ with a basis $a_{1}, \cdots, a_{r}$ such that $\beta\left(a_{k}\right) \leqq n$ for all $k$. B $\left.y b\right)$, every vector in $U$ is a sum of linearly independent vectors $u_{i}$ each satisfying $\beta\left(u_{i}\right) \leqq n$. We complete the proof by proving, by induction on $k$, the statement $P_{k}:$ If $u_{1}, \cdots, u_{k}$ are linearly independent vectors of $U$ each satisfying

$$
\beta\left(u_{i}\right) \leqq n, \text { then } \beta\left(\sum_{i=1}^{k} u_{i}\right) \leqq 2 m n .
$$

$P_{k}$ is true by a) for $k=1,2, \cdots, 2 m$. Assume now that $k>2 m$ and that $P_{i}$ holds for all $i<k$. Let $u_{1}, \cdots, u_{2 m}, \cdots, u_{k}$ be linearly independent vectors of $U$ each satisfying $\beta\left(u_{i}\right) \leqq n$. For $1 \leqq i \leqq m$ let $y_{i}=u_{2 i-1}+u_{2 i}$ so that

$$
u=u_{1}+\cdots+u_{k}=y_{1}+\cdots+y_{m}+u_{2 m+1}+\cdots+u_{k} \text {. }
$$

Then $y_{1}, \cdots, y_{m}$ are linearly independent and so $\left\langle y_{1}, \cdots, y_{m}\right\rangle$ has dimension $m$ and therefore contains a non-zero vector $w$ such that $\beta(w) \leqq n$. The element $w$ is expressible as $\sum_{i=1}^{m} \alpha_{i} y_{i}$ where not all $\alpha_{i}$ are zero. We may assume, without loss in generality, that $\alpha_{1} \neq 0$ so that

Thus

$$
y_{1}=\alpha_{1}^{-1} w-\sum_{i=2}^{m} \alpha_{1}^{-1} \alpha_{i} y_{i}
$$

$$
u_{1}+u_{2}=\alpha_{1}^{-1} w-\sum_{i=2}^{m} \alpha_{1}^{-1} \alpha_{i}\left(u_{2 i-1}+u_{2 i}\right) .
$$

Substituting for $u_{1}+u_{2}$ we can express $u$ as a linear combination of $w, u_{3}, \cdots, u_{k}$ and these are linearly independent vectors. $P_{k}$ now follows from the induction hypothesis.

We note that, if $U$ is a subspace of the $T$-space $V$, then the rank of $x$ on $U$ is a function $\beta(x)$ which satisfies a) and b) of Lemma 2.7. We have stated the lemma in more generality than necessary in order to be able to use it in a subsequent paper. 


\section{Proper closed subsets of $\mathfrak{I}$}

Definition. The set of $T$-spaces which have a totally singular subspace of codimension at most $r$ obviously forms a closed subset of $\mathfrak{I}$. We call this subset the $r$ th hyperlayer and denote it by $\mathfrak{I}(r)$. Obviously, $\mathfrak{I}(1) \subset \mathfrak{I}(2) \subset \cdots$ and $\bigcup_{r=1}^{\infty} \mathfrak{I}(r)=\mathfrak{I}$. The main theorem of this section is that a closed subset of $\mathfrak{I}$ which is not equal to $\mathfrak{I}$ itself is contained in one of the hyperlayers.

LemMa 3.1. Let $V$ be a $T$-space such that $V_{1}^{r} V$ and let $U$ be any totally singular subspace of $V$. Then every $r^{2}$-dimensional subspace of $V$ contains a non-zero element whose rank on $U$ is less than $12 r^{2}$.

Proof. Suppose, if possible, that $X$ is an $r^{2}$-dimensional subspace of $V$ every non-zero element of which has rank on $U$ at least $12 r^{2}$. By Lemma 1.2, $X$ has a totally singular subspace $P$ of dimension $r$. Applying Lemma 2.6 with $U$ in place of $V_{0}$ we see that (taking $\left.n=1\right) V$ has a sub-T-space isomorphic to $V\left(1, r, P, x^{(1)}, \cdots, x^{(r)}\right)$ where $x^{(1)}, \cdots, x^{(r)}$ is any basis for $P$. But, since $P$ is totally singular, this sub- $T$-space is isomorphic to $V_{1}^{r}$. This contradiction proves the lemma.

LeMma 3.2. Suppose that $V$ is a $T$-space such that $V_{1}^{r} V$ and let $U$ be any totally singular subspace of $V$. Then there exist subspaces $V_{0}$ of $V$ and $U_{0}$ of $U$ such that

a) $\left[V: V_{0}\right] \leqq r^{2}$

b) $\left[U: U_{0}\right] \leqq 144 r^{8}$

c) $\left(V_{0}, U_{0}, U_{0}\right)=0$

d) $U \leqq V_{0}$.

Proof. For any $x \in V$ define $\beta(x)$ to be the rank of $x$ on $U$. Then

1) $\beta(x+y) \leqq \beta(x)+\beta(y)$ for all $x, y \in V$

2) $\beta(\lambda x)=\beta(x)$ for all $x \in V$ and $\lambda \in F-\{0\}$.

Moreover, by the previous lemma, every $r^{2}$-dimensional subspace of $V$ contains a non-zero element $x$ such that $\beta(x)<12 r^{2}$. We can apply Lemma 2.7 to obtain a subspace $V_{0}$ of $V$ such that $\left[V: V_{0}\right] \leqq r^{2}$ and $\beta(x)<24 r^{4}$ for all $x \in V_{0}$. In particular, $V_{0}$ satisfies a).

Since every element of $V_{0}$ has rank on $U$ less than $24 r^{4}$, Lemma 2.3 guarantees the existence of a subspace $U_{0}$ of $U$ satisfying b) and c). Finally, since $\left(U, U_{0}, U_{0}\right)$ $=0$, we can replace $U+V_{0}$ by $V_{0}$ and satisfy d).

Lemma 3.3. Let $V$ be a $T$-space such that $V_{1}^{r} V$. Then $V$ has a totally singular subspace of codimension at most $\left(2.144 r^{8}+2 r^{2}+1\right)^{2}-1$. 
ProOF. Let $U$ be a totally singular subspace of maximal dimension and take $V^{*}$ so that $U \oplus V^{*}=V$. If the lemma is false then $\operatorname{dim} V^{*} \geqq\left(2.144 r^{8}+2 r^{2}+1\right)^{2}$ and so, by Lemma $1.2, V^{*}$ has a totally singular subspace $W$ of dimension $2.144 r^{8}+2 r^{2}+1$. By the previous lemma there exist subspaces $U_{0} \leqq U$, $V_{0}^{(1)} \leqq V, W_{0} \leqq W, V_{0}^{(2)} \leqq V$ such that

a) $\left[V: V_{0}^{(1)}\right] \leqq r^{2}$ and $\left[V: V_{0}^{(2)}\right] \leqq r^{2}$

b) $\left[U: U_{0}\right] \leqq 144 r^{8}$ and $\left[W: W_{0}\right] \leqq 144 r^{8}$

c) $\left(V_{0}^{(1)}, U_{0}, U_{0}\right)=0$ and $\left(V_{0}^{(2)}, W_{0}, W_{0}\right)=0$

d) $U \leqq V_{0}^{(1)}$ and $W \leqq V_{0}^{(2)}$.

If we put $A=U_{0} \cap V_{0}^{(2)}$ and $B=W_{0} \cap V_{0}^{(1)}$ then a) implies that $\left[U_{0}: A\right] \leqq r^{2}$ and $\left[W_{0}: B\right] \leqq r^{2}$. By b), $[U: A] \leqq 144 r^{8}+r^{2}$ and $[W: B]$ $\leqq 144 r^{8}+r^{2}$. Moreover, since $A \cap B=0, \operatorname{dim}(A+B)=\operatorname{dim} A+\operatorname{dim} B$ and it follows that

$$
\operatorname{dim}(A+B) \geqq \operatorname{dim} U-\left(144 r^{8}+r^{2}\right)+\operatorname{dim} W-\left(144 r^{8}+r^{2}\right)=\operatorname{dim} U+1 .
$$

However, if $C=V_{0}^{(1)} \cap V_{0}^{(2)}, C$ contains both $A$ and $B$ and, by c), $(C, A, A)$ $=(C, B, B)=0$ and hence $A+B$ is totally singular. This contradicts the choice of $U$.

THEOREM 3.4. A closed subset of $\mathfrak{I}$ which does not contain every $T$-space is contained in one of the hyperlayers.

Proof. Let $\mathfrak{X}$ be a closed subset of $\mathfrak{I}$ which is not equal to $\mathfrak{T}$. By Theorem 2.2 there exists $n$ such that $V_{1}^{n} \neq V$ for all $V \in \mathfrak{X}$. It follows from Lemma 3.3 that $\mathfrak{X} \subseteq \mathfrak{I}(m)$ where $m=\left(2.144 n^{8}+2 n^{2}+1\right)^{2}-1$.

\section{The main theorem}

In this section we prove the main theorem on $T$-spaces - that $(\mathfrak{I}, \preccurlyeq)$ is a partially well-ordered set. As a corollary of the proof we obtain a description of all closed subsets of $\mathfrak{T}$.

Definition. Let $P$ be an $r$-dimensional $T$-space with an $s$-dimensional sub- $T$-space $Q$. Let $\mathfrak{F}(P, Q)$ denote the closure of the set of $T$-spaces $V$ which satisfy the following conditions:

a) $V=S \oplus \widetilde{P}$ where $S$ is totally singular and $P$ is isomorphic to $\widetilde{P}$ in an isomorphism $x \rightarrow \tilde{x}$ which carries $Q$ to $\tilde{Q}$

b) $(\tilde{Q}, S, S)=0$

c) $(\widetilde{Q}, \widetilde{Q}, S)=0$. 
We note that $\mathfrak{F}(P, Q)$ depends not only on $P$ and $Q$ but also on the particular way in which $Q$ is embedded in $P$.

The unique $T$-space $V$ which satisfies a), b), c) and the further conditions

d) $\tilde{P}=\widetilde{Q} \oplus \tilde{R}$ where $\tilde{R}$ corresponds to a subspace $R$ of $P$ in the isomorphism and $R$ has a basis $x^{(s+1)}, \cdots, x^{(r)}$

e) $(\tilde{Q}, \widetilde{R}, S)=0$

f) $\widetilde{R} \oplus S=V\left(n, r-s, \widetilde{R}, \tilde{x}^{(s+1)}, \cdots, \tilde{x}^{(r)}\right)$ in that $S$ has a basis $\left\{a_{i}^{(j)}, b_{i}^{(j)} \mid 1 \leqq i \leqq n, s+1 \leqq j \leqq r\right\}$ and the basic products of $\tilde{R} \oplus S$ resemble those of the definition of $V\left(n, r, P, x^{(1)}, \cdots, x^{(r)}\right)$ in an obvious way, is denoted by $S\left(n, r, s, P, Q, R, x^{(s+1)}, \cdots, x^{(r)}\right)$. This also depends on the embeddings of $Q$ and $R$ into $P$.

It is easy to see that $\left\{S\left(n, r, s, P, Q, R, x^{(s+1)}, \cdots, x^{(r)}\right)\right\}_{n=1}^{\infty}$ is an ascending chain in $(\mathfrak{I}, \preccurlyeq)$.

LEMMA 4.1. In the notation of the previous definition let $V$ be a $T$-space satisfying $a), b$ ) and c). Assume that a subspace $R$ of $P$ and basis $x^{(s+1)}, \cdots, x^{(r)}$ is chosen so that d) holds. Then, for some $n$,

$$
V \preccurlyeq S\left(n, r, s, P, Q, R, x^{(s+1)}, \cdots, x^{(r)}\right) .
$$

Proof. Let $x^{(1)}, \cdots, x^{(s)}$ be a basis for $Q$. Consider a $T$-space $\bar{\nabla}$ isomorphic to $S\left(n, r, s, P, Q, R, x^{(s+1)}, \cdots, x^{(r)}\right)$. We may suppose that it has the following structure:

1) $\bar{V}=\bar{Q} \oplus \bar{R} \oplus S$ where $P$ is isomorphic to $\bar{P}=\bar{Q} \oplus \bar{R}$ in an isomorphism $x \mapsto \bar{x}$ which carries $Q$ to $\bar{Q}$ and $R$ to $\bar{R}$; in particular, for $1 \leqq i, j, k \leqq r$,

$$
\left(\bar{x}^{(i)}, \bar{x}^{(j)}, \bar{x}^{(k)}\right)=\left(x^{(i)}, x^{(j)}, x^{(k)}\right)=\left(\tilde{x}^{(i)}, \tilde{x}^{(j)}, \tilde{x}^{(k)}\right)
$$

2) $S$ is totally singular

3) $(\bar{Q}, S, S)=0$

4) $(\bar{P}, \bar{P}, S)=0$

5) $S$ has a basis $\left\{a_{\imath}^{(j)}, b_{i}^{(j)} \mid 1 \leqq i \leqq n, s+1 \leqq j \leqq r\right\}$ where $\left(\bar{x}^{(i)}, a_{l}^{(j)}, b_{m}^{(k)}\right)$ $=\delta_{j k} \delta_{i j} \delta_{l m}, \quad$ for $s+1 \leqq i, j, k \leqq r$ and $1 \leqq l, m \leqq n$ and $\left(\bar{x}^{(i)}, a_{l}^{(j)}, a_{m}^{(k)}\right)$ $=\left(\bar{x}^{(i \prime}, b_{l}^{(j)}, b_{m}^{(k)}\right)=0$, for $s+1 \leqq i, j, k \leqq r$ and $1 \leqq l, m \leqq n$.

We may assume that $V$ is non-singular; for if we can embed the non-singular part of $V$ into $\bar{V}$ then the singular elements may be embedded into $\bar{S}$ if necessary by taking $n$ to be larger. It is enough to map the basis elements of $V$ into $\bar{V}$ in such a way that the trilinear form is preserved, for then we can extend the map to a homomorphism which, by Lemma 2.1 , will be a monomorphism.

Let $s_{1}, \cdots, s_{c}$ be a basis for $\mathrm{S}$ and let $U_{1}, \cdots, U_{p}$ be all the subsets $\left\{s_{i}, s_{j}\right\}$ with $i<j$. Take $n$ to be any integer not less than $p+r$. For each $k=1,2, \cdots, p$ define a map $\Delta_{k}: U_{k} \rightarrow S$ by $s_{i} \Delta_{k}=a_{k}^{(s+1)}+\cdots+a_{k}^{(r)}$ and $s_{j} \Delta_{k}=\left(\tilde{x}^{(s+1)}, s_{i}, s_{j}\right) b_{k}^{(s+1)}+$ $\cdots+\left(\tilde{x}^{(r)}, s_{i}, s_{j}\right) b_{k}^{(r)}$ where $i<j$ and $\left\{s_{i}, s_{j}\right\}=U_{k}$. 
We now define a linear transformation $\beta$ of $V$ into $\bar{V}$ as follows. For $1 \leqq i \leqq c$,

$$
\begin{gathered}
s_{i} \beta=s_{i 1}+\cdots+s_{i p}+\sum_{\substack{m=1 \\
n=s+1}}^{\substack{n=r \\
m=r}}\left(\tilde{x}^{(m)}, \tilde{x}^{(n)}, s_{i}\right) a_{p+m}^{(n)} \\
\text { where } s_{i k}= \begin{cases}s_{i} \Delta_{k} & \text { if } s_{i} \in U_{k} \\
0 & \text { otherwise. }\end{cases}
\end{gathered}
$$

For $1 \leqq k \leqq r$, $\tilde{x}^{(k)} \beta=\bar{x}^{(k)}+\sum_{l=s+1}^{r} \gamma_{k l} b_{k+p}^{(l)} \quad$ where $\gamma_{k l}=1$ if $k<l$ and 0 if $k \geqq l$.

To show that $\beta$ is a monomorphism we have to verify
A) $\left(\tilde{x}^{(i)} \beta, \tilde{x}^{(j)} \beta, \tilde{x}^{(k)} \beta\right)=\left(\tilde{x}^{(i)}, \tilde{x}^{(j)}, \tilde{x}^{(k)}\right)$ for all $1 \leqq i, j, k \leqq r$,
B) $\left(s_{t} \beta, s_{j} \beta, s_{k} \beta\right)=\left(s_{i}, s_{j}, s_{k}\right)$ for all $1 \leqq i, j, k \leqq c$,
C) $\left(\tilde{x}^{(u)} \beta, s_{v} \beta, s_{w} \beta\right)=\left(\tilde{x}^{(u)}, s_{v}, s_{w}\right)$ for all $1 \leqq u \leqq r$ and $1 \leqq v<w \leqq c$,
D) $\left(\tilde{x}^{(u)} \beta, \tilde{x}^{(v)} \beta, s_{w} \beta\right)=\left(\tilde{x}^{(u)}, \tilde{x}^{(v)}, s_{w}\right)$ for all $1 \leqq u<v \leqq r, 1 \leqq w \leqq c$.

A) $\left(\tilde{x}^{(i)} \beta, \tilde{x}^{(j)} \beta, \tilde{x}^{(k)} \beta\right)$

$$
\begin{aligned}
& =\left(\bar{x}^{(i)}+\sum_{l=s+1}^{r} \gamma_{i l} b_{i+p}^{(l)}, \bar{x}^{(j)}+\sum_{l=s+1}^{r} \gamma_{j l} b_{j+p}^{(l)}, \bar{x}^{(k)}+\sum_{l=s+1}^{r} \gamma_{k l} b_{k+p}^{(l)}\right) \\
& =\left(\bar{x}^{(i)}, \bar{x}^{(j)}, \bar{x}^{(k)}\right)=\left(\tilde{x}^{(i)}, \tilde{x}^{(j)}, \tilde{x}^{(k)}\right) .
\end{aligned}
$$

B) This follows since both sides are equal to zero.

C) $\left(\tilde{x}^{(u)} \beta, s_{v} \beta, s_{w} \beta\right)$

$$
\begin{aligned}
& =\left(\bar{x}^{(u)}+\sum_{l=s+1}^{r} \gamma_{u l} b_{u+p}^{(l)}, s_{v 1}+\cdots+s_{v p}+\sum_{\substack{m=1 \\
n=s+1}}^{\substack{n=r \\
m=r}}\left(s_{v}, \tilde{x}^{(m)}, \tilde{x}^{(n)}\right) a_{p+m}^{(n)},\right. \\
& \left.s_{w 1}+\cdots+s_{w p}+\sum_{\substack{m=1 \\
n=1}}^{\substack{n=r \\
m=r}}\left(s_{w}, x^{(m)}, x^{(n)}\right) a_{p+m}^{(n)}\right) \\
& =\left(\bar{x}^{(u)}, s_{v 1}+\cdots+s_{v p}, s_{w 1}+\cdots+s_{w p}\right) \text {, from the definition of } \bar{V} \text {, and so } \\
& \left(\tilde{x}^{(u)} \beta, s_{v} \beta, s_{w} \beta\right)= \begin{cases}0 & \text { if } u>s \\
\sum_{i=s+1}^{r}\left(\bar{x}^{(u)}, s_{v i}, s_{w i}\right) & \text { if } u \leqq s\end{cases}
\end{aligned}
$$

by definition of $\bar{V}$. Now, if $\left\{s_{v}, s_{w}\right\}=U_{k}$, then $\left(\bar{x}^{(u)}, s_{v i}, s_{w i}\right)$ is non-zero only when 
both $s_{v i}$ and $s_{w i}$ are non-zero and this occurs only when both $s_{v}$ and $s_{w}$ belong to $U_{\imath}$, i.e. when $i=k$. Thus,

$$
\begin{aligned}
\left(\tilde{x}^{(u)} \beta, s_{v} \beta, s_{w} \beta\right) & = \begin{cases}0 & \text { if } u \leqq s \\
\left(\bar{x}^{(u)}, s_{v k}, s_{w k}\right) & \text { if } u>s\end{cases} \\
& = \begin{cases}0 & \text { if } u \leqq s \\
\left(\bar{x}^{(u)}, s_{v} \Delta_{k}, s_{w} \Delta_{k}\right) & \text { if } u>s\end{cases} \\
& = \begin{cases}0 & \text { if } u \leqq s \\
\left(\bar{x}^{(u)}, \sum_{l=s+1}^{r} a_{k}^{(l)}, \sum_{l=s+1}^{r}\left(x^{(l)}, s_{v}, s_{w}\right) b_{k}^{(l)}\right. & \text { if } u>s .\end{cases}
\end{aligned}
$$

Using again the definition of $\bar{V}$ we have

$$
\begin{aligned}
& \left(\tilde{x}^{(u)} \beta, s_{v} \beta, s_{w} \beta\right)= \begin{cases}0 & \text { if } u \leqq s \\
\left(\bar{x}^{(u)}, a_{k}^{(u)},\left(\tilde{x}^{(u)}, s_{v}, s_{w}\right) b_{k}^{(u)}\right) & \text { if } u>s\end{cases} \\
& = \begin{cases}0 & \text { if } u \leqq s \\
\left(\tilde{x}^{(u)}, s_{v}, s_{w}\right) & \text { if } u>s\end{cases} \\
& =\left(\tilde{x}^{(u)}, s_{v}, s_{w}\right) \quad \text { f } 1 \leqq u \leqq r \text { since } \quad\left(\tilde{x}^{(u)}, s_{v}, s_{w}\right)=0 \\
& \text { if } u \leqq s . \\
& \text { D) }\left(\tilde{x}^{(u)} \beta, \tilde{x}^{(v)} \beta, s_{w} \beta\right)=\left(\bar{x}^{(u)}+\sum_{l=s+1}^{r} \gamma_{u l} b_{u+p}^{(l)}, \bar{x}^{(v)}+\sum_{l=s+1}^{r} \gamma_{v l} b_{v+p}^{(l)}\right. \text {, } \\
& \left.s_{w 1}+\cdots+s_{w p}+\sum_{\substack{m=1 \\
n=s+1}}^{\substack{n=r \\
m=r}}\left(\tilde{x}^{(m)}, \tilde{x}^{(n)}, s_{w}\right) a_{p+m}^{(n)}\right) \\
& =\left(\bar{x}^{(u)}, \sum_{l=s+1}^{r} \gamma_{v l} b_{v+p}^{(l)}, \sum_{\substack{m=1 \\
n=s+1}}^{\substack{n=r \\
m=r}}\left(\tilde{x}^{(m)}, \tilde{x}^{(n)}, s_{w}\right) a_{p+m}^{(n)}\right) \\
& +\left(\sum_{l=s+1}^{r} \gamma_{u l} b_{u+p}^{(l)}, \bar{x}^{(v)}, \sum_{\substack{m=1 \\
n=s+1}}^{\substack{n=r \\
m=r}}\left(\tilde{x}^{(m)}, \tilde{x}^{(n)}, s_{w}\right) a_{p+m}^{(n)}\right) \\
& =\left(\bar{x}^{(u)}, \sum_{l=s+1}^{r} \gamma_{v l} b_{v+p}^{(l)}, \sum_{n=s+1}^{r}\left(\tilde{x}^{(v)}, \tilde{x}^{(n)}, s_{w}\right) a_{v+p}^{(n)}\right)
\end{aligned}
$$




$$
\begin{aligned}
&+\left(\sum_{l=s+1}^{r} \gamma_{u l} b_{u+p}^{(l)}, x^{(v)}, \sum_{n=s+1}^{r}\left(\tilde{x}^{(u)}, \tilde{x}^{(n)}, s_{w}\right) a_{u+p}^{(n)}\right) \\
&=\left\{\begin{array}{l}
0 \text { if } 1 \leqq u<v \leqq s \\
\left(\gamma_{u v} b_{u+p}^{(v)}, \bar{x}^{(v)},\left(\tilde{x}^{(u)}, \tilde{x}^{(v)}, s_{w}\right) a_{u+p}^{(v)}\right) \text { if } 1 \leqq u \leqq s<v \leqq r \\
\left(\bar{x}^{(u)}, \gamma_{v u} b_{v+p}^{(u)},\left(\tilde{x}^{(v)}, \tilde{x}^{(u)}, s_{w}\right) a_{v+p}^{(u)}\right) \\
+\left(\gamma_{u v} b_{u+p}^{(v)}, \bar{x}^{(v)},\left(\tilde{x}^{(u)}, \tilde{x}^{(v)}, s_{w}\right) a_{u+p}^{(v)}\right) \text { if } s<u<v \leqq r
\end{array}\right. \\
&=\left\{\begin{array}{l}
0 \text { if } 1 \leqq u<v \leqq s \\
\left(\tilde{x}^{(u)}, \tilde{x}^{(v)}, s_{w}\right) \text { if } 1 \leqq u \leqq r \text { and } s<v \leqq r \text { since } \gamma_{u v}=1
\end{array}\right. \\
& \text { and } \gamma_{v u}=0\left(\tilde{x}^{(u)}, \tilde{x}^{(v)}, s_{w}\right) \text { if } 1 \leqq u<v \leqq r \text { since }\left(\tilde{x}^{(u)}, \tilde{x}^{(v)}, s_{w}\right)=0 \\
& \text { if } 1 \leqq u<v \leqq s .
\end{aligned}
$$

Thus, A), B), C) and D) are all true and hence $\beta$ is a monomorphism of $V$ into $\bar{V}$. Therefore $V$ is isomorphic to a sub-T-space of $\bar{V}$ and hence $V \preccurlyeq S\left(n, r, s, P, Q, R, x^{(s+1)}, \cdots, x^{(r)}\right)$ as required.

The next lemma is, in some sense, a converse of Lemma 4.1, for it gives a condition under which a $T$-space of $\mathfrak{F}(P, Q)$ has a subspace which is isomorphic to $S\left(n, r, s, P, Q, R, x^{(s+1)}, \cdots, x^{(r)}\right)$. We shall use both lemmas at the end of the proof of Lemma 4.3 to deduce that $\mathfrak{F}(P, Q)$ is the closure of a certain set of $T$-spaces.

LEMMA 4.2. Let $V$ be a T-space which satisfies conditions a), b) and $c)$ of the definition of $\mathfrak{F}(P, Q)$. Assume that a subspace, $R$, of $P$ and a basis, $x^{(s+1)}, \cdots, x^{(r)}$, of $R$ is chosen so that $\left.d\right)$ holds. If every element of $\tilde{R}-\{0\}$ has rank on $S$ at least $8 n^{2} r^{2}+6 r^{2}$ then $S$ has a subspace $S_{1}$ such that

$$
\widetilde{P} \oplus S_{1} \text { is isomorphic to } S\left(n, r, s, P, Q, R, x^{(s+1)}, \cdots, x^{(r)}\right) .
$$

ProOF. Let $S_{0}=S \cap \operatorname{Ann}(\tilde{Q}, \widetilde{R})$ so that $\left[S: S_{0}\right] \leqq r s \leqq r^{2}$. Hence, by Lemma 1.3, every element of $\tilde{R}-\{0\}$ has rank on $S_{0}$ at least $8 n^{2} r^{2}+6 r^{2}-2 r^{2}$ $=8 n^{2} r^{2}+4 r^{2}$. We may now apply Lemma 2.6 to $\widetilde{R} \oplus S_{0}$ (with $\widetilde{R}$ in place of $P$ and $S_{0}$ in place of $V_{0}$ ). This yields a subspace, $S_{1}$, of $S_{0}$ which satisfies

i) $\left(\tilde{Q}, \tilde{R}, S_{1}\right)=0$

ii) $\tilde{R} \oplus S_{1}=V\left(n, r-s, \tilde{R}, \tilde{x}^{(s+1)}, \cdots, \tilde{x}^{(r)}\right)$.

It follows that $\tilde{P} \oplus S_{1}$ is isomorphic to $S\left(n, r, s, P, Q, R, x^{(s+1)}, \cdots, x^{(r)}\right)$.

LEMMA 4.3. Let $\mathfrak{X}=\left\{V_{i}\right\}_{i=1}^{\infty}$ be an infinite set of $T$-spaces whose closure is not $\mathfrak{I}$ itself. Then there exists a subset of $\mathfrak{X}$ whose closure is $\mathfrak{F}(P, Q)$ for some $T$-space $P$ and sub-T-space $Q$ of $P$. 
Proof. We shall prove this lemma by successively replacing $\mathfrak{X}$ by suitable subsets until we obtain one with the right property.

Since $\operatorname{cl} \mathfrak{X} \neq \mathfrak{I}$, Theorem 3.4 implies that, for some integer $k, \mathfrak{X} \subseteq \mathfrak{I}(k)$. Each $T$-space $V_{i}$ of $\mathfrak{X}$ has, therefore, a decomposition $A_{i} \oplus U_{i}$ where $\operatorname{dim} A_{i} \leqq k$ and $U_{i}$ is totally singular. Since $F$ is finite, there are only a finite number of possibilities for $A_{i}$ (up to isomorphism of $T$-spaces) and one of these possibilities must occur infinitely often. Hence, if we replace $\mathfrak{X}$ by a suitable infinite subset, we may assume that $A_{i} \cong A$ for all $i$.

For each $i$ and $x \in A$ write $x(i)$ for the element of $A_{i}$ which corresponds to $x$ in the isomorphism of $A$ with $A_{i}$. Write $r_{i}(x)$ for the rank of $x(i)$ on $U_{i}$.

We choose a subset, $C$, of $A$ maximal under inclusion with respect to the following property: there exists a subsequence $n_{1}, n_{2}, \cdots$ of $1,2, \cdots$ such that $r_{n_{i}}(x) \rightarrow \infty$ as $i \rightarrow \infty$ for all $x \in C$. Since $A$ is finite and we allow the possibility that $C$ may be empty, $C$ certainly exists. Let $B=A-C$. We shall show that $B$ is a subspace of $A$. Let $b \in B$. If $r_{n_{i}}(b)$ is not bounded as $i \rightarrow \infty$ there exists a subsequence $m_{1}, m_{2}, \cdots$ of $n_{1}, n_{2}, \cdots$ such that $r_{m_{i}}(b) \rightarrow \infty$ as $i \rightarrow \infty$ and hence $r_{m_{i}}(x) \rightarrow \infty$ as $i \rightarrow \infty$ for all $x \in\{b\} \cup C$ and this contradicts the maximality of $C$. Thus $B$ consists precisely of those elements $x \in A$ for which $r_{n_{i}}(x)$ is bounded as $i \rightarrow \infty$. It follows from the relations

$$
r_{n}(x+y) \leqq r_{n}(x)+r_{n}(y), r_{n}(\alpha x)=r_{n}(x) \text { if } \alpha \neq 0 \text { and } r_{n}(0)=0
$$

that $B$ is a subspace. Replacing $\mathfrak{X}$ by $\left\{V_{n_{i}}\right\}_{i=1}^{\infty}$ we may assume that $r_{n}(x)$ is bounded as $n \rightarrow \infty$ if $x \in B$ and $r_{n}(x) \rightarrow \infty$ as $n \rightarrow \infty$ if $x \in A-B$.

Let $b_{1}, \cdots, b_{t}$ be a basis for $B$ and let

$$
B_{i}=\left\langle b_{1}(i), \cdots, b_{t}(i)\right\rangle \leqq A_{i} .
$$

There exist integers $h_{1}, \cdots, h_{t}$ such that $r_{i}\left(b_{j}\right) \leqq h_{j}$ for all $i$. Thus, for all $i$ and for $j=1,2, \cdots, t$, the subspace

$$
D_{i j}=\left\{x \in U_{i} \mid\left(b_{j}(i), x, U_{i}\right)=0\right\}
$$

has codimension at most $h_{j}$ in $U_{i}$. Let $D_{i}=\bigcap_{j=1}^{t} D_{i j}$. Then, for each $i, D_{i}$ has codimension at most $h=\Sigma_{j=1}^{t} h_{j}$ in $U_{i}$ and $\left(B_{i}, D_{i}, U_{i}\right)=0$. Let $S_{i}=D_{i}$ $\cap \operatorname{Ann}\left(B_{i}, B_{i}\right)$ so that, by Lemma 1.1, $\left[D_{i}: S_{i}\right] \leqq t^{2}$ and hence $\left[U_{i}: S_{i}\right] \leqq t^{2}+h$. Moreover, $\left(B_{i}, B_{i}, S_{i}\right)=\left(B_{i}, S_{i}, U_{i}\right)=0$.

Now, for each $i$, choose a subspace, $T_{i}$, such that $U_{i}=S_{i} \oplus T_{i}$. Since $\operatorname{dim} T_{i} \leqq t^{2}+h$, there are only a finite number of possibilities for $T_{i}$ (up to isomorphism of $T$-spaces) and so one of these possibilities must occur infinitely often. By replacing $\mathfrak{X}$ by a suitable subsequence we may assume that $T_{i} \cong T$ for all $i$. 
As before, for each $i$ and $t \in T$, write $t(i)$ for the element of $T_{i}$ which corresponds to $t$ in the isomorphism of $T$ with $T_{r}$. Extend the basis $b_{1}, \cdots, b_{t}$ of $B$ to a basis $b_{1}, \cdots, b_{t}, \cdots, b_{u}$ of $A$ and let $t_{1}, \cdots, t_{v}$ be a basis for $T$.

For each $i$ consider the basic products formed with the elements $b_{1}(i)$, $\cdots, b_{u}(i), t_{1}(i), \cdots, t_{v}(i)$ (which form a basis for $A_{i} \oplus T_{\imath}$ ). Those basic products of the form $\left(b_{1}(i), b_{m}(i), b_{n}(i)\right)$ and $\left(t_{1}(i), t_{m}(i), t_{n}(i)\right)$ are independent of $i$, while there are only a finite number of possibilities for the $u \times u \times v$ array $\left(b_{l}(i), b_{m}(i)\right.$, $\left.t_{n}(i)\right)$ and for the $u \times v \times v$ array $\left(b_{l}(i), t_{m}(i), t_{n}(i)\right)$. Thus, as usual, we may replace $\mathfrak{X}$ by a suitable subsequence so that the $u \times u \times v$ array $\left(b_{l}(i), b_{m}(i), t_{n}(i)\right)$ is independent of $i$ and then replace (the new) $\mathfrak{X}$ by a subsequence so that the $u \cdot v \cdot v$ array $\left(b_{l}(i), t_{m}(i), t_{n}(i)\right)$ is independent of $i$. Then all the sub- $T$-spaces $A_{i} \oplus T_{i}$ are isomorphic to some $T$-space of the form $A \oplus T$ in isomorphisms in which $A_{i}$ corresponds to $A, B_{i}$ to $B$ and $C_{i}$ to $C$.

Let $P=A \oplus T, Q=B \oplus T$ and choose $R \leqq A$ so that $B \oplus R=A$. Write $P_{i}, Q_{i}, R_{\imath}$ for the sub-T-spaces of $A_{i} \oplus T_{i}$ which correspond to $P, Q, R$. Then, for each $i$, the following conditions hold.

a) $V_{i}=S_{i} \oplus P_{i}, S_{i}$ totally singular

b) $\left(Q_{i}, S_{i}, S_{i}\right)=0$, because $\left(T_{i}, S_{i}, S_{i}\right)=\left(B_{i}, S_{i}, S_{i}\right)=0$

c) $\left(Q_{i}, Q_{i}, S_{i}\right)=0$, because $\left(T_{i}, T_{i}, S_{i}\right)=\left(B_{i}, B_{i}, S_{\imath}\right)=\left(T_{i}, B_{i}, S_{i}\right)=0$.

Thus, $\mathfrak{X} \subseteq \mathfrak{F}(P, Q)$. Moreover, $P=Q \oplus R$. Since $R-\{0\} \subseteq A-B$ it follows that $r_{i}(x) \rightarrow \infty$ as $i \rightarrow \infty$ for all $x \in R-\{0\}$. However, since $\left[U_{i}: S_{i}\right]$ is bounded independently of $i$ by $t^{2}+h$, it follows that, for all $x \in R-\{0\}$, the rank of $x(i)$ on $S_{i}$ tends to infinity with $i$. Thus, by Lemma 4.2 , clæ contains $S(n, r, s, P, Q, R$, $x^{(s+1)}, \cdots, x^{(r)}$ ) for every $n$ (where $r=\operatorname{dim} P, s=\operatorname{dim} Q$ and $x^{(s+1)}, \cdots, x^{(r)}$ is a basis for $R)$. Hence, clł $=\mathfrak{F}(P, Q)$ by Lemma 4.1.

THEOREM 4.4. $(\mathfrak{I}, \preccurlyeq)$ is a partially well-ordered set.

Proof. Since $\mathfrak{I}$ consists of finite dimensional vector spaces it is obvious that $(\mathfrak{I}, \preccurlyeq)$ satisfies the minimum condition. Let $\mathfrak{X}$ be an arbitrary infinite subset of $\mathfrak{I}$. We wish to show that there exist distinct $T$-spaces $U, V \in \mathfrak{X}$ such that $U \preccurlyeq V$. If $\mathfrak{I}$ contains an infinite ascending chain $\mathfrak{Y}$ such that $\mathrm{cl} \mathfrak{X}=\mathrm{cl} \mathfrak{Y}$ then we can take any $U \in \mathfrak{X}$, find $Y_{1} \in \mathfrak{Y}$ with $U \preccurlyeq Y_{1}$, find $Y_{2} \in \mathfrak{Y}$ with $Y_{1} \preccurlyeq Y_{2}$ and $Y_{1} \neq Y_{2}$ and then find $V \in \mathfrak{X}$ with $Y_{2} \preccurlyeq V$. If $\mathrm{cl} \mathfrak{X}=\mathfrak{I}$ we can, using Theorem 2.2, take $\left\{V_{1}^{n}\right\}_{n=1}^{\infty}$ as $\mathfrak{Y}$. If $\operatorname{cl} \mathfrak{X} \neq \mathfrak{I}$ then, by Lemma 4.3 , we can suppose that $\mathrm{cl} \mathfrak{X}=\mathfrak{F}(P, Q)$ and then, using Lemma 4.1 , take $\left\{S\left(n, r, s, P, Q, R, x^{(s+1)}, \cdots, x^{(r)}\right)\right\}_{n=1}^{\infty}$ as $\mathfrak{Y}$.

Another obvious consequence of Lemma 4.3 is that every closed subset of $\mathfrak{I}$ is the union of the $\mathfrak{F}(P, Q)$ which it contains together with finitely many other $T$-spaces. 


\section{Varieties of groups}

In this section we survey the main steps in proving the following theorems

THEOREM 5.1. If $m$ is any integer coprime to 3 then $A_{m} B_{3}$ is hereditarily finitely based.

THEOREM 5.2. $B_{6}$ is hereditarily finitely based.

We begin by indicating how Theorem 5.2 can be deduced from Theorem 5.1. The fact, due to Hall in [2], that $B_{6}$ is locally finite implies that it is generated by its critical groups. Now, a critical group of exponent 6 has 2-length and 3-length equal to 1 (see [3]) and from this it follows that every critical group of exponent 6 belongs either to $A_{2} B_{3}$ or $B_{3} A_{2}$ and, as $B_{3} A_{2}$ is a Cross variety, it suffices to prove that $A_{2} B_{3}$ is hereditarily finitely based. But this follows from Theorem 5.1.

To prove Theorem 5.1 the first step is to use some arguments due to Higman in [4] to reduce to the case where $m$ is a prime $p$ not equal to 3 .

In this case a critical group $G$ of $A_{p} B_{3}-B_{3}$ is a split extension $G=N T$ of a normal elementary abelian $p$-subgroup $N$ and a group $T$ of exponent 3 . Because $N$ is a faithful irreducible module for $T$ it follows that $T$ has cyclic centre $Z$. By counting conjugacy classes in $T$ and $T / Z$ it can be shown that $T$ has exactly two absolutely irreducible faithful representations and then, by studying automorphisms of $T$, one can prove that, up to similarity, there is only one possibility for the representation of $T$ on $N$. Thus, $T$ determines $G$ up to isomorphism.

If we regard $T / T^{\prime}$ as a vector space over $G F(3)$ then the commutator function $[x, y, z]$ induces an alternating trilinear form on $T / T^{\prime}$. It is possible to show that this form uniquely determines $T$. Thus, the critical group $G$ determines and is determined by a certain $T$-space $V_{G}$.

Now the Kovács and Newman theory of minimal representations is applied (see Chapter 5 of [5]) and from this it follows that the subvarieties of $A_{p} B_{3}$ are in $1-1$ correspondence with the factor closed sets of critical groups in $A_{p} B_{3}$. Thus, to prove Theorem 5.1 it suffices to prove that these factor closed sets of critical groups satisfy the minimal condition under inclusion; or, equivalently, that the set of critical groups is partially well-ordered under involvement. It is necessary, therefore, to consider conditions which guarantee that one critical group $H$ is a factor of another critical group $G$. One can show that a sufficient condition for this is that $V_{H} \preccurlyeq V_{G}$ and then Theorem 4.4 completes the proof.

\section{References}

[1] M. D. Atkinson, D. Phil. thesis, (Oxford, 1970).

[2] M. Hall, 'Solution of the Burnside problem for exponent 6', Illinois.J. Math. 2 (1958), 764-786. 
[3] G. Higman and P. Hall, 'The $p$-length of a $p$-soluble group and reduction theorems for Burnside's problem', Proc. London Math. Soc. (3) 7 (1956), 1-42.

[4] G. Higman, 'The orders of relatively free groups'. Proc. Internat. Conf. Theory of Groups, Aust. Nat. Univ. Canberra August 1965, 153-165, (Gordon and Breach, New York, 1967).

[5] Hanna Neumann: Varieties of groups, (Berlin - Heidelberg - New York, Springer - Verlag (1967).

The Queen's College, Oxford, England

Present address:

Department of Computing Mathematics

University College

Cardiff, Wales 